\title{
Nouveaux taxa de micro-algues dulçaquicoles pour le Burkina Faso (Afrique de l'Ouest): I- Chlorophyta
}

\author{
Frédéric ZONGO ${ }^{1 *}$, Bilassé ZONGO ${ }^{1}$, Joseph I. BOUSSIM ${ }^{1}$ et Alain COUTÉ ${ }^{2}$ \\ ${ }^{1}$ Université de Ouagadougou, Unité de Formation et de Recherche en Sciences de la Vie et de la Terre, \\ Laboratoire de Biologie et Écologie Végétales, 09 BP 848 Ouagadougou 09, Burkina Faso. \\ ${ }^{2}$ Muséum Nationale d'Histoire Naturelle, Département RDDM, Paris, France. \\ *Auteur correspondant, E-mail: gulb.zongo@yahoo.fr
}

\begin{abstract}
RÉSUMÉ
L'étude taxonimique des micro-algues échantillonnées dans le réservoir de Bagré (Burkina Faso, Afrique de l'Ouest), a permis d'inventorier et de décrire 70 nouveaux taxa (Chlorophyta), qui se répartissent dans 11 familles, 29 genres. Parmi les familles, les Desmidiaceae sont les plus abondantes avec 44,3\% puis viennent les Oocystaceae $(15,7 \%)$, les Scenedesmaceae en égalité avec les Closteriaceae $(11,4 \%)$, les Dictyosphaeriaceae (4,3\%), les Chlorococcaceae qui sont en égalité avec les Radiococcaceae et Volvocaceae $(2,9 \%)$ et enfin les Hydrodictyaceae, les Gloeocystaceae et les Phacotaceae avec chacune 1,4\%. Ces nouveaux taxa ont été observés et décrits grâce à un microscope photonique. Avec 70 nouvelles espèces inventoriées pour le Burkina Faso, le réservoir de Bagré peut être considéré comme un milieu favorable au développement de certaines espèces de Chlorophyta.
\end{abstract}

(C) 2008 International Formulae Group. All rights reserved.

Mots clés : Micro-algues, Chlorophyta, taxinomie, réservoir de Bagré, Burkina Faso

\section{INTRODUCTION}

Au Burkina Faso, à l'opposé des plantes supérieures qui ont fait l'objet de nombreux travaux d'inventaires depuis 1899 , les recherches concernant la microflore et, plus spécialement, les micro-algues sont à un stade embryonnaire (comme d'ailleurs dans les autres régions d'Afrique). A notre connaissance, il n'existe que quelques études partielles sur ce thème. Parmi celles-ci, on peut citer les études de Gauthier-Lièvre (1964, 1965) qui se sont intéressées à la taxinomie des Zygnemataceae africaines. Au Burkina Faso, ces travaux se sont déroulés dans la partie ouest du pays. Les études de Roman (1977, 1979), plus ponctuelles, se sont limitées uniquement aux genres. Ces travaux ont porté sur le phytoplancton des retenues d'eau alimentant la ville de Ouagadougou : à savoir le réservoir $n^{\circ} 3$ de Ouagadougou et le réservoir de Loumbila. Les travaux de
Hiddinga (1981) sur les Desmidiaceae; ceux de Zongo (1994) et Zongo \& Guinko (1999a) qui ont aussi été entrepris dans le réservoir $\mathrm{n}^{\circ} 3$ de la ville de Ouagadougou; ceux de Zongo et Guinko (1999b, 1999c, 2000) qui ont porté sur la flore algale du pays. Beaucoup d'autres travaux ont encore été menés au Burkina Faso sur la flore algale. Nous pouvons citer entre autres les travaux de Da et al. (2004) qui ont porté sur un nouveau genre de Péridiniales (Dinophyta) d'eau douce (le genre Bagredinium) découvert dans le réservoir de Bagré au Burkina Faso; ceux de Zerbo (2004) qui ont décrit le phytoplancton du réservoir de Loumbila (Burkina Faso); ceux de Zongo et al. (2006) qui concernent une nouvelle espèce appartenant au genre Strombomonas découvert dans le réservoir de Bagré; ceux de Zongo (2007) qui se sont intéressés au phytoplancton du canal du parc urbain Bangr-wéoogo de Ouagadougou. 
La présente étude des micro-algues échantillonnées dans le réservoir de Bagré nous a permis d'inventorier, d'illustrer et de décrire un certain nombre de nouveaux taxa appartenant à l'embranchement des Chlorophyta pour le Burkina Faso. Elle vient en complément à celles réalisées par les différents auteurs ci-dessus cités. Elle permettra à cet effet de contribuer à une meilleure connaissance de la microflore algale du Burkina faso.

\section{MATERIEL ET METHODES}

A partir du matériel dont nous disposions, une analyse des paramètres physico-chimiques de l'eau et une récolte d'échantillons de micro-algues ont été faites.

\section{Site de récoltes}

Le réservoir de Bagré, dont la mise en eau a eu lieu en 1991, est construit sur la rivière Nakambé (ex Volta Blanche), affluent du Nazinon (ex Volta Rouge). Il est situé à environ 150 kilomètres au Sud-Est de
Ouagadougou, capitale du Burkina Faso (Afrique de l'Ouest) (coordonnées du site: $11^{\circ} 27^{\prime} \mathrm{N}$ et $\left.0^{\circ} 30^{\prime} \mathrm{W}\right)$. En période de hautes eaux (fin de la saison des pluies), sa superficie atteint 25.000 hectares et la profondeur au niveau du barrage est d'environ $40 \mathrm{~m}$. Pendant la saison sèche (basses eaux), la surface se réduit à 8.500 hectares avec une profondeur maximale avoisinant $25 \mathrm{~m}$.

Ce réservoir a une double vocation à savoir, d'une part, la production d'électricité et, d'autre part, l'irrigation.

Il a été l'objet d'une étude entre 1995 et 1998, dans le cadre d'un projet hollandais (projet RUG-Écologie) qui visait à réaliser un suivi de la qualité de ses eaux et à enrichir la connaissance de la microflore algale du Burkina Faso.

$\mathrm{Au}$ cours de cette période, du matériel biologique a été échantillonné dans deux sites au niveau du village de Foungou et de celui de Bagré (Figure 1).

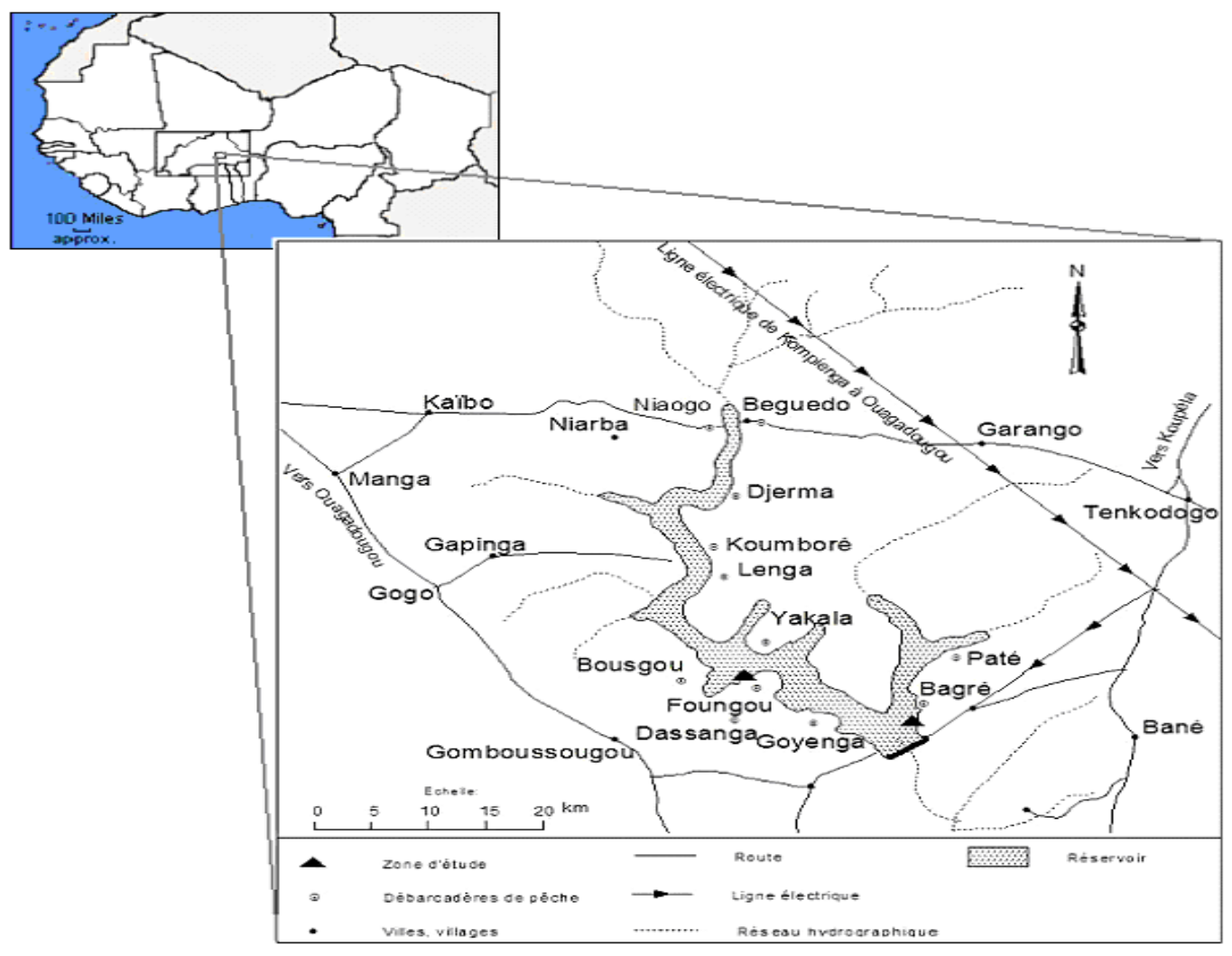

Figure 1: Localisation géographique du réservoir de Bagré au Burkina Faso. En encart, situation du Burkina Faso sur le continent Africain. 
Techniques d'analyses physico-chimiques

Les analyses physico-chimiques de l'eau ont été faites dans la période Mars 1997Février 1998 (une année). Ce qui nous a permis d'apprécier globalement la qualité de l'eau dans le réservoir de Bagré jusqu'à la fin de notre étude.

Lors de chaque récolte, certains paramètres physico-chimiques ont été mesurés in situ. Des échantillons d'eau ont été prélevés et conservés dans une glacière afin de maintenir la qualité de l'eau pour des analyses ex situ au laboratoire.

Pour les paramètres physico-chimiques mesurés et étudiés :

in situ, la conductivité a été mesurée à l'aide d'un conductimètre de marque WTW, l'oxygène et la température avec un oxygènemètre de type $\mathrm{O}_{2}$-meter $\mathrm{CG} 867$, le $\mathrm{pH}$ avec un pHmètre HI 8424 microcomputer pHmeter et la transparence avec un disque de Secchi ;

ex situ (au laboratoire, deux heures après les prélèvements), les taux de nitrates, de nitrites, d'ammonium, d'azote Kjedhal, des orthophosphates, du phosphate total et de la chlorophylle $a$, à l'aide d'un spectrophotomètre Hach de type DR 2000.

Techniques de récoltes et de préparation des échantillons de micro-algues

Les micro-algues ont été récoltées en utilisant un filet à plancton de $50 \mu \mathrm{m}$ de vide de maille. Elles ont été fixées immédiatement avec une solution aqueuse de formaldéhyde à $8 \%$ et conservées à l'obscurité pour réduire les risques de dépigmentation trop rapide. Les prélèvements sur le site ont été faits deux fois dans le mois (la moitié et la fin de chaque mois). La référence de chaque échantillon prend en compte le site et la date de prélèvement. Exemple: éch. F 7/97= échantillon prélevé à Foungou à la fin du mois de juillet de l'an 1997 ; B 5/97 = échantillon prélevé à Bagré à la fin du mois de mai de l'an 1997. Lorsque l'année citée se termine par un prime, cela signifie que l'échantillon a été prélevé le 15 du mois. Exemple : éch. F 5/97'.

\section{Observations et identifications des espèces}

Les observations et les dessins ont été effectués à l'aide d'un microscope photonique de type Wild M 20 équipé d'une chambre claire. En effet, à partir de chaque échantillon de base, un sous échantillon est prélevé et observé au microscope. Au total 91 échantillons ont été observés. Les espèces rencontrées sont représentées à l'aide d'un tube à dessins dont dispose le microscope photonique.

Les espèces rencontrées et dessinées sont ensuite identifiées grâce à des clés d'identification. Les clés utilisées sont: Bourrelly (1990) et Huber-Pestalozzi (1961).

\section{RÉSULTATS \\ Données écologiques}

Le tableau 1 donne les valeurs mensuelles des paramètres mesurés au niveau de Foungou de mars 1997 à février 1998.

Les moyennes des valeurs des paramètres physico-chimiques mesurés montrent que les eaux du réservoir de Bagré au niveau de Foungou sont alcalines $(\mathrm{pH}$ : 8,31 ), faiblement minéralisées (conductivité : $92 \mu \mathrm{S} \mathrm{cm}^{-1}$ ) et eutrophes (azote Kjedhal : 2,16 $\mathrm{mg} \mathrm{L}^{-1}$; phosphate total : $0,44 \mathrm{mg} \mathrm{L}^{-1}$; chlorophylle a: $4,44 \mu \mathrm{g} \mathrm{\textrm {L } ^ { - 1 }}$; transparence : $0,49 \mathrm{~m})$.

Les écarts types calculés à partir paramètres montrent une faible variation des différents paramètres ci-dessus cités au cours de l'année, toutes saisons confondues. Les valeurs de ces paramètres s'éloignent donc faiblement des moyennes calculées. Les valeurs des moyennes ainsi que des écarts types sont données dans le tableau 1.

Description des nouveaux taxa (Chlorophyta) découverts dans le réservoir de Bagré pour le Burkina Faso

Comme mentionné ci-dessus, les taxa observés; dessinés et décrits dans cette étude ont été réalisés dans des échantillons prélevés au niveau de deux sites durant les trois années (1995-1998). Pour chaque espèce, sont donnés : la ou les synonymies entre griffes et les caractères morphologiques observés par nous-même.

La répartition biogéographique des micro-algues étant jusque là embryonnaire, l'aire d'extension des espèces correspond actuellement à l'aire de distribution des algologues. Nous donnons alors chaque fois que possible, la distribution de chaque espèce décrite. 
Tableau 1: Valeurs mensuelles des paramètres mesurés

\begin{tabular}{|c|c|c|c|c|c|c|c|c|}
\hline Mois & $\underset{\mathrm{Cemp}}{\text { Tem. }}$ & $\begin{array}{l}\text { Cond. } \\
\mu S \text { cm }^{-1}\end{array}$ & pH & $\begin{array}{c}\text { Oxygène } \\
\text { mg l}^{-1}\end{array}$ & $\begin{array}{c}\text { Transp. } \\
\text { M }\end{array}$ & $\begin{array}{c}\text { Azote } \\
\text { Kjedahl } \\
\text { mgN L }^{-1}\end{array}$ & $\begin{array}{l}\text { Phosphate total } \\
\text { mgPO }_{4}{ }^{3-} \mathrm{L}^{-1}\end{array}$ & $\begin{array}{c}\text { Chl «a» } \\
\mu \mathrm{g} / \mathrm{l}\end{array}$ \\
\hline Mars 1997 & 30,20 & 90,00 & 7,88 & 6,70 & 0,60 & 0,39 & 0,38 & 1,84 \\
\hline Avril & 31,40 & 94,00 & 8,42 & 7,70 & 0,70 & 1,65 & 0,18 & 0,69 \\
\hline Mai & 31,30 & 95,00 & 8,45 & 7,10 & 0,60 & 2,85 & 0,22 & VM \\
\hline Juin & 30,30 & 109,00 & 8,57 & 7,40 & 0,60 & 0,75 & 0,21 & 1,10 \\
\hline Juillet & 28,80 & 112,00 & 8,37 & 6,60 & 0,40 & 9,60 & 2,84 & 1,38 \\
\hline Août & 30,00 & 95,00 & 7,64 & 6,30 & 0,30 & 3,30 & 0,15 & 4,60 \\
\hline Sept. & 30,30 & 86,00 & 7,90 & 6,30 & 0,20 & 2,40 & 0,08 & 5,52 \\
\hline Octobre & 32,20 & 76,00 & 8,03 & 7,50 & 0,25 & 1,80 & 0,21 & 5,52 \\
\hline Nov. & 29,80 & 87,00 & 8,73 & 8,30 & 0,40 & 0,75 & 0,15 & 16,56 \\
\hline Déc. & 24,40 & 83,00 & 8,52 & 8,90 & 0,40 & 1,05 & 0,16 & 3,07 \\
\hline Jan. 1998 & 30,00 & 87,00 & 9,19 & 9,00 & 0,60 & 0,90 & 0,16 & 3,01 \\
\hline Fév. & 28,50 & 91,00 & 8,03 & 6,90 & 0,45 & 0,45 & 0,11 & 5,52 \\
\hline Moy. & 29,77 & 92,00 & 8,31 & 7,39 & 0,49 & 2,16 & 0,44 & 4,44 \\
\hline Ecart types & 11,03 & 31,93 & 3,34 & 3,04 & 0,14 & 0,84 & 0,26 & 0,58 \\
\hline
\end{tabular}

Temp. = température ; Cond. = conductivité Transp. = transparence; $\mathrm{Chl} \ll \mathrm{a} »=$ chlorophylle $a$; VM = Valeur manquante

Le résumé de la liste des espèces par famille et par embranchement est présenté dans le tableau 2.

\section{Embranchement des Chlorophyta}

Famille des Chlorococcaceae

Tetraedron caudatum (Corda) Hansg. var. incisum (Lagerh.) Brunth.

fig. 3, éch. : B 12/97'

[Syn. : Polyedrium caudatum Corda, $P$. tetragonum Reinsch, $P$. trigonum fo. pentagona (Reinsch) Rabenh., Tetraedron caudatum var. longispinum Lemm., T. caudatum var. punctatum Lagerh.]

Les cellules, pentagonales, ont des côtés concaves dont un montre une profonde incision. Les angles sont munis chacun d'un court aiguillon.

Dimensions : cellules de 20-25 $\mu \mathrm{m}$ de diamètre sans les épines.

Distribution: cosmopolite. En Afrique : Afrique du Sud, Maroc, Mozambique, Tchad.

Tetraedron cruciatum (Wall.) G. S. West fig. 4, éch. : F 2/98

[Syn.: Tetraedron gracile (Reinsch) Hansg.]

Les cellules, cruciformes, sont prolongées aux angles par des appendices creux bifurqués. Le plaste pariétal, a un pyrénoïde.

Dimensions: cellules de 20-30 $\mu \mathrm{m}$ de diamètre.
Distribution: cosmopolite. En Afrique : Ouganda, Tchad.

\section{Famille des Dictyosphaeriaceae}

Botryococcus braunii Kütz.

$$
\text { fig. 5, éch. : F.2/96 }
$$

[Syn.: Botryococcus giganteus Reinsch, Gomphosphaeria aurantiaca Bleish sensu Dang, Ineffigiata neglecta W. et G.S. West]

Les cellules, obovoïdes à piriformes, sont disposées radialement à la périphérie des colonies, et presque complètement enveloppées par la gelée coloniale. Chaque cellule a un plaste pariétal pourvu d'un pyrénoïde difficilement visible localisé dans la partie étroite de la cellule.

Distribution: cosmopolite. En Afrique : Afrique du Sud, Côte d'Ivoire, Égypte, Éthiopie, Kenya, Madagascar, Malawi, Mali, Maroc, Ouganda, République Démocratique du Congo, Sénégal, Sierra Léone, Soudan, Tanzanie, Tchad, Zambie, Zimbabwé.

\section{Dimorphococcus lunatus A. Braun fig.6, éch. : F.10/97 \\ [Syn.: Dictyosphaerium reniforme Bulnh.]}

La colonie, globuleuse, est composée de nombreuses cellules réniformes unies entre elles par les fragments de la paroi maternelle. Le plaste pariétal recouvrant la plus grande 
part de la paroi cellulaire, renferme un pyrénoïde.

Dimensions : cellules de 7-12 x 4-6 $\mu \mathrm{m}$.

Distribution: cosmopolite. En Afrique: Afrique du Sud, Algérie, Kenya, Malawi, Madagascar, Mali, Mozambique, Ouganda, République Démocratique du Congo, Soudan, Tanzanie, Tchad, Zambie, Zimbabwé.

Westella botryoides (West) De Wild. fig. 7, éch. : F.9/95

Les colonies, sont composées de cellules sphériques disposées en croix par groupe de huit. Ces groupes sont connectés les uns aux autres par les débris de la paroi maternelle. Le chloroplaste est unique, cupuliforme, avec un seul pyrénoïde.

Dimensions : cellules de 6-7 $\mu \mathrm{m}$ de diamètre. Distribution : cosmopolite. En Afrique : Côte d'Ivoire, Mozambique.

\section{Famille des Hydrodictyaceae}

Pediastrum biradiatum Meyen var. longecornutum Gutw.

fig. 8, éch.F 2/97

Le cénobe, plat, circulaire, perforé, est composé de quatre cellules à partie basale incurvée. Ces cellules sont en contact avec leur base seulement. Elles ont chacune deux lobes formés par une incision profonde. Chaque lobe est en forme de V. La paroi cellulaire est lisse.

Dimensions : cellules de 15-17 x 10-12 $\mu \mathrm{m}$. Distribution: cosmopolite. En Afrique : Mozambique.

\section{Famille des Oocystaceae}

\section{Chodatella quadriseta Lemm.} fig. 9 éch. : B 5/95

[Syn.: Chodatella quadriseta var. brevicaudata Skv., Lagerheimia quadriseta (Lemm.) G.M. Smith]

Les cellules, ellipsoïdales à subglobuleuses, ont des pôles arrondis ornés chacun de deux longues soies.

Dimensions : cellules de 8-10 x 3-6 $\mu \mathrm{m}$, soies de $14-17 \mu \mathrm{m}$.

Distribution: cosmopolite. En Afrique : Kenya, Maroc, Ouganda, Tanzanie, Tchad.

Chodatella subsalsa Lemm. fig. 10, éch. : B 5/95
[Syn. : Chodatella octocantha (Lemm.) Ley, Ch. maxima Hortob., Lagerheimia subsalsa Lemm., L. ciliata var. subsalsa (Lemm.) Playf.]

Les cellules ellipsoïdales, ont des pôles arrondis, ornés chacun de 4 longues soies.

Dimensions : cellules de 9-12 x 5-7 $\mu \mathrm{m}$, soies longues de 13-15 $\mu \mathrm{m}$.

Distribution: subcosmopolite. En Afrique : Afrique du Sud, Algérie, Maroc, Ouganda, République Démocratique du Congo, Tanzanie, Tchad, Zambie.

Monoraphidium griffithii (Brek.)
Kom.-Legner
B2/97

Les cellules fusiformes, droites, sont graduellement amincies aux extrémités et se terminent en pointe. On observe un chloroplaste pariétal allongé.

Dimensions : cellules de 23-80 x 2-5 $\mu \mathrm{m}$.

Distribution: subcosmopolite. En Afrique: Côte d'Ivoire, Malawi, Ouganda, République Démocratique du Congo, Rwanda, Sénégal, Tchad.

\section{Echinosphaerella limnetica G.M. Smith fig. 12, éch.: F 7/95 [Syn. : Tetraedron schmidlei (Schröd.) Lemm., T. hastatum Schmidle, Treubaria limnetica (G.M. Smith) Fott et Kovac.]}

Cette espèce, unique dans le genre présente les caractéristiques propres au genre. Les cellules ont des diamètres cependant supérieurs à celui de l'espèce-type $(10 \mu \mathrm{m})$ et les soies sont également plus longues et plus larges à leurs bases que celles de l'espèce type (20-25 $\mu \mathrm{m}$ de longueur; largeur à leur base de $3 \mu \mathrm{m}$ ).

Dimensions : cellules de 14-16 $\mu \mathrm{m}$ de diamètre, soies longues de 30-33 $\mu$ m et larges à leur base de $5 \mu \mathrm{m}$.

Distribution: Europe; Asie; Amérique du nord et du sud

\section{Kirchneriella obtusa (Korsh.) Kom.}

fig. 13, éch.: B 2/97

Les cellules, largement fusiformes, arrondies aux extrémités, un peu courbées, sont groupées par 8 en colonies gélatineuses. Elles renferment chacune, un plaste pariétal muni d'un pyrénoïde.

Dimensions : cellules de 11-14 x 6-8 $\mu \mathrm{m}$. 
Distribution : espèce généralement des régions tempérées, mais déjà décrite en Afrique, au Sénégal, par Compère (1991).

\section{Monoraphidium indicum Hind.}

fig. 14, éch. : F 4/97

Les cellules, fusiformes, étroites, sont 37 fois plus longues que larges. Elles sont sigmoïdes aiguës aux extrémités. Le plaste pariétal, est sans pyrénoïde.

Dimensions : cellules de 70-75 x $2 \mu \mathrm{m}$.

Distribution : Afrique (Sénégal, Tchad)

\section{Nephrocytium limneticum (G.M. Smith) G.M. Smith fig. 15, éch. : F 4/95}

[Syn. : Nephrocytium limneticum (G.M. Smith) Skuja]

Les cellules, réniformes ou en forme de saucisse, sont largement arrondies aux extrémités. Elles forment des colonies gélatineuses de 4 ou 8 cellules.

Dimensions : cellules de 15-20 x 8-12 $\mu \mathrm{m}$

Distribution: subcosmopolite. En Afrique : Tchad, Zambie.

\section{Nephrocytium lunatum W. West} fig. 16, éch.B 2/97

Les cellules, en forme de croissant, pointues aux extrémités, sont groupées par 4 ou 8 en colonies enveloppées dans la paroi maternelle. Dimensions : cellules de 6-15 x 3-4 $\mu \mathrm{m}$ Distribution: cosmopolite. En Afrique : Afrique du Sud, Algérie, Côte d'Ivoire, Kenya, République Démocratique du Congo, Soudan, Tchad, Zambie, Zimbabwé.

Nephrocytium obesum W. et G.S. West fig. 17, éch.: B 6/97' Les cellules, hémisphériques ou ellipsoïdales, sont largement arrondies aux extrémités et regroupées par 4 en colonies enveloppées dans la paroi maternelle.

Dimensions : cellules de 24-27 x 14-15 $\mu \mathrm{m}$. Distribution: cosmopolite. En Afrique : Mozambique, Tchad, Zambie.

\section{Oocystis crassa Wittr.} fig. 18, éch. : F 8/95

[Syn.: Oocystis solitaria Wittr., $O$. solitaria var. apiculata (W. West) Printz, O. solitaria var. rupestris (Kirchn.) Hansg.]
Les cellules, ellipsoïdales, solitaires ou groupées en colonies de 4, au moment de la sporulation, ont une paroi pourvue d'un nodule en mamelon à chaque pôle. Elles renferment 5-10 plastes pariétaux pourvus chacun d'un pyrénoïde.

Dimensions : cellules de 16-18 x 12-13 $\mu \mathrm{m}$

Distribution: cosmopolite. En Afrique : Afrique du Sud, Madagascar, Mali, Maroc, Mozambique, République Démocratique du Congo, Tanzanie, Tchad, Zambie.

Treubaria euryacantha (Schmidle) Korsch. fig. 19, éch. : F 11/97

Les cellules, globuleuses, sont pourvues de quatre aiguillons coniques hyalins disposés dans un plan. On observe, par cellule, un à trois plastes renfermant chacun un pyrénoïde. Dimensions : cellules de $10-13 \mu \mathrm{m}$ de diamètre, aiguillons de 20-30 $\mu \mathrm{m}$ de longueur. Distribution : cosmopolite.

\section{Famille des Radiococcaceae}

\section{Coenochloris pseudoquadriguloides}

Tell fig. 20, éch. : F 8/97
Les colonies sont composées de 32 cellules ovales ou réniformes, en 8 groupes de 4 cellules chacun. Les fragments des parois maternelles sont présents dans la gelée commune. Le plaste pariétal renferme un pyrénoïde.

Dimensions : cellules de 5-7 x 3-4 $\mu$ m.

\section{Coenochloris pyrenoidosa Korsch.}

fig. 21, éch. : B 12/96

Les colonies sont composées de 32 cellules subsphériques, en 8 groupes de 4 cellules chacun. Les fragments des parois maternelles sont présents dans la gelée commune. Le plaste pariétal à un pyrénoïde.

Dimensions: cellules subsphériques de 5-10 $\mu \mathrm{m}$ de diamètre.

\section{Famille des Scenedesmaceae \\ Actinastrum aciculare Playf. fo. africanum Compère \\ fig. 22, éch. : B 12/97'}

Les cellules, étroitement ovoïdes-lancéolées, longuement et finement pointues au sommet, arrondies à la base, sont groupées en cénobes 
étoilés de 4-8 cellules. Le plaste pariétal est pourvu d'un pyrénoïde.

Dimensions : cellules de 20-28 x 2-3 $\mu \mathrm{m}$.

Distribution: Afrique du Sud, Sénégal, Soudan, Tchad.

Actinastrum hantzschii Lagerh. var. fluviatile Schröder

fig. 23, éch.: B 12/97,

Les cellules, étroitement cylindriquesfusiformes, rétrécies et aiguës aux deux extrémités, sont groupées en cénobes étoilés de 8 cellules; une cellule n'étant pas sur le même plan que les autres, donc pas visible au niveau de notre représentation. Le plaste pariétal est pourvu d'un pyrénoïde.

Dimensions : cellules de 14-15 x 2-3 $\mu \mathrm{m}$.

Distribution: subcosmopolite. En Afrique: Tchad.

Coelastrum cambricum Arch. var. intermedium (Bohl.) G.S.West fig. 24, éch. : F 8/95

[Syn.: Coelastrum indicum Turn., $C$. intermedium (Bohl.) Korsch., $C$. pulchrum var. intermedius Bohl.]

Les colonies sphériques sont composées de cellules subsphériques à excroissance apicale arrondie (apparaissant comme un épaississement de la paroi). Les cellules sont unies entre elles par des appendices courts et larges.

Dimensions : cellules de 10-13 $\mu \mathrm{m}$ de diamètre.

Distribution: cosmopolite. En Afrique : Afrique du Sud, Côte d'Ivoire, Guinée, Libye, Malawi, Mali, Maroc, Ouganda, Sénégal, Tanzanie Tchad, Zimbabwé.

\section{Coelastrum}

Korsch.

pseudomicroporum fig. 25, éch.: F.8/97

Les colonies sphériques sont composées de cellules sphériques à subsphériques unies les unes aux autres par de courts appendices connectifs. Les espaces intercellulaires sont relativement petits.

Dimensions : cellules de 14-15 $\mu \mathrm{m}$ de diamètre.

Distribution : Europe. En Afrique : Tchad. Crucigenia crucifera (Wolle) Collins fig. 26, éch. : B 12/97'

[Syn. : Crucigeniella crucifera (Wolle) Kom., C. cruciata Schmidle, $C$. reniforme Swir.]
Les cénobes, rhomboïdaux, ont quatre cellules réniformes. Chaque cellule renferme un plaste pariétal à un pyrénoïde.

Dimensions : cellules de 10-12 x 4-5 $\mu \mathrm{m}$

Distribution: cosmopolite. En Afrique : Tchad, Zimbabwé

Scenedesmus balatonicus Hortob. fig. 27, éch. : B 6/97

Les cénobes sont composés de 4-8 cellules cylindriques à oblongues-ellipsoïdales, disposées en série linéaire et présentant des méats étroits, linéaires, à elliptiqueslancéolées.

Dimensions : cellules de 11-14 x 5-7 $\mu \mathrm{m}$.

Distribution: Europe, Asie. En Afrique : Tchad.

$$
\begin{gathered}
\text { Scenedesmus obtusus Meyen fo. } \\
\text { ecornis (Ehr.) Compère } \\
\text { fig. 28, éch. : F 7/95 }
\end{gathered}
$$

[Syn.: Scenedesmus quadricaudatus var. ecornis Ehr., Sc. quadricauda var. ecornis (Her.) Ralfs, Sc. ecornis (Ehr.) Chod.]

Les cénobes sont composés de 4 cellules ovoïdes à ellipsoïdales, arrondies aux pôles et disposées en une série linéaire.

Distribution: cosmopolite. En Afrique: Afrique du Sud, Algérie, Angola, Égypte, Éthiopie, Guinée Bissau, Kenya, Libye, Madagascar, Malawi, Maroc, Mozambique, Ouganda, République Centrafricaine, République Démocratique du Congo, Sénégal, Soudan, Sud-Ouest Africain, Tanzanie, Tchad, Zambie, Zimbabwé.

Tetrallantos lagerheimii Teil. fig.29, éch. : B 11/97'

Espèce formée de quatre cellules présentant les mêmes caractéristiques que le genre.

Dimensions : cellules de 20-22 x $5 \mu \mathrm{m}$.

Distribution: cosmopolite. En Afrique: Afrique du Sud, Côte d'Ivoire, Mozambique, Tchad.

\section{Famille des Gloeocystaceae}

Gloeocystis ampla (Kütz.) Rabenh. fig. 30, éch. : B 12/96

[Syn. : Chlamydocapsa ampla (Kütz.) Fott, Sphaerellocystis ampla (Kütz.) Novak.] Les cellules, ovoïdes à subsphériques, sont entourées d'une large gaine gélatineuse 
individuelle, groupées en une colonie gélatineuse. On observe un plaste en urne pourvu d'un pyrénoïde basal.

Dimensions : cellules de 10-13 x 8-10 $\mu \mathrm{m}$.

Distribution: cosmopolite. En Afrique : Afrique du Sud, Algérie, Angola, Maroc, Sierra Léone, Tchad, Zimbabwé.

\section{Famille des Phacotaceae}

\section{Wislouchiella planctonica Skv.} fig. 31, éch. : F 10/97'

Espèce présentant toutes les caractéristiques du genre.

Dimensions : logettes de $38-40$ x 22-24 $\mu \mathrm{m}$

Distribution: espèce connue jusque là uniquement de Mandchourie et des États Unis.

\section{Famille des Volvocaceae}

\section{Gonium pectorale Müll.} fig. 32, éch.: F 8/97

[Syn. : Gonium helveticum Perty] Les colonies plates, subquadrangulaires, sont composées de 16 cellules ovoïdes ou subsphériques; disposées en deux séries. Chacune des cellules est pourvue d'une gaine gélatineuse connectée à celles des autres par de courts prolongements gélatineux. Les plastes en urne sont pourvus d'un pyrénoïde basal. La différence avec G. formosum repose sur le fait que l'ensemble des cellules et leur gaine individuelle est entourée d'une gelée commune. Les deux flagelles que dispose chaque cellule sortent de la gelée commune Dimensions : cellules de 10-13 $\mu \mathrm{m}$ de diamètre.

Distribution: cosmopolite. En Afrique : Afrique du Sud, Algérie, Égypte, Guinée, Madagascar, Mozambique, République Démocratique du Congo, Soudan, Tanzanie, Tchad, Zambie.

\section{Gonium sociale (Duj.) Warming} fig. 33, éch. : F 2/97

[Syn.: Gonium tetras A. Br., Tetragonium lacustre W. et G.S. West] Les colonies plates, quadrangulaires, sont composées de quatre cellules ovoïdes, subsphériques, entourées chacune d'une gaine gélatineuse reliée à celles des autres par de courts prolongements. Chaque cellule est munie de deux flagelles plus ou moins longs.
Le chloroplaste, en urne, est pourvu d'un pyrénoïde.

Dimensions : cellules de 10-12 $\mu \mathrm{m}$.

Distribution : cosmopolite.

\section{Famille des Closteriaceae}

Closterium acerosum (Schrank) Ehr. var. borgei (Borge) Krieger

fig. 34, éch.: B 12/97

La cellule 10 fois plus longue que large, est rétrécie brusquement dans la région apicale et légèrement concave au niveau de la marge externe (dorsale). Les pôles sont coniques. La paroi est lisse et brunâtre. Le chloroplaste, en cinq crêtes longitudinales, renferme 10 pyrénoïdes axiaux en une série.

Dimensions : cellule de 400 x $40 \mu \mathrm{m}$.

Distribution : cosmopolite

Closterium acerosum (Schrank) Ehr. var. minus Hantzsch

fig. 35, éch.: F 9/97

Les cellules, 9 à 12 fois plus longues que larges, ont une marge externe courbe et une marge interne rectiligne. La paroi est brunâtre et lisse.

Dimensions : cellules de 138-315 x 14-27 $\mu \mathrm{m}$. Distribution: cosmopolite. En Afrique : Maroc, Tchad

Closterium acutum Bréb. ex Ralfs var. variabile (Lemm.) Krieger fig. 36, éch. : 2/97

Les cellules, de 24 à 25 fois plus longues que larges, sont fortement et souvent irrégulièrement courbées. La paroi est lisse et le chloroplaste renferme 2-3 pyrénoïdes.

Dimensions : cellules de 73-123 x 3-5 $\mu \mathrm{m}$.

Distribution : cosmopolite. En Afrique : Côte d'Ivoire, Mali, Mozambique, Soudan, Tchad, Zambie.

Closterium dianae Ralfs var. brevius (Petk.) Krieg. fig. 37, éch. : F 9/97 La cellule, 7,6 fois plus longue que large, courbée, a une marge interne renflée dans sa partie médiane. Elle est tronquée obliquement et pourvue d'un pore à chaque pôle. La paroi est lisse, brunâtre, et le chloroplaste a 6 pyrénoïdes disposés en une série.

Dimensions : cellule de 100 × $13 \mu \mathrm{m}$.

Distribution: cosmopolite. En Afrique : Guinée, Tchad. 


\section{Closterium limneticum Lemm.}

fig. 38, éch. : F 5/97

La cellule, plus longue que large, presque rectiligne est un peu courbée aux extrémités. La paroi est lisse et le chloroplaste a 6 pyrénoïdes.

Dimensions : cellule de 126 × $3 \mu \mathrm{m}$. Cette espèce présente des caractéristiques presque similaires à celles de $\mathrm{Cl}$. gracile (Bréb.) Ralfs. Il y a une confusion réelle entre ces deux taxons.

Distribution: cosmopolite. En Afrique : Afrique du Sud, Algérie, Guinée, Madagascar, Mali, Mozambique, Ouganda, République Démocratique du Congo, Sierra Léone, SudOuest Africain, Tanzanie, Tchad, Zambie, Zimbabwé.

\section{Closterium parvulum Näg.} fig. 39, éch. : F. 9/97

Les cellules, courbes, plus longues que larges, sont atténuées et plus ou moins aiguës aux pôles. La paroi est lisse et le chloroplaste a 2 pyrénoïdes.

Dimensions : cellules de 85-87 x 10-12 $\mu \mathrm{m}$.

Distribution: cosmopolite. En Afrique : Afrique du Sud, Algérie, Côte d'Ivoire, Égypte, Guinée, Libye, Ouganda, Madagascar, Malawi, Maroc, Mozambique, République Démocratique du Congo, Sierra Léone, Soudan, Tanzanie, Tchad, Zambie.

\section{Closterium praelongum Bréb.} fig. 40 , éch.: F $8 / 97$

La cellule, 20 fois plus longue que large, a une courbure régulière. Les marges sont parallèles dans la région médiane et modérement atténuées dans les régions apicales. La paroi brunâtre, est finement striée (apparemment lisse).

Dimensions : cellule de 600 × $30 \mu \mathrm{m}$

Distribution : cosmopolite. En Afrique : Côte d'Ivoire, République Démocratique du Congo, Tchad.

\section{Closterium toxon W. West}

fig. 41, éch.: B.12/97

La cellule, 21 fois plus longue que large, est rectiligne dans la zone médiane avec des marges parallèles. La zone apicale, courbée, a un pôle tronqué. La paroi lisse, est brunâtre. Le chloroplaste renferme 10 pyrénoïdes disposés en série.

Dimensions : cellules de $350 \times 12 \mu \mathrm{m}$
Distribution: cosmopolite. En Afrique : Tchad.

\section{Famille des Desmidiaceae}

Cosmarium aculeatum Forster var. africanum Couté et Rousselin fig. 42, éch. : F 8/95

La cellule, plus longue que large, présente une constriction médiane peu profonde. Le sinus est largemment ouvert et les hémisomates sont ovales. La paroi est ornée d'aiguillons courts et aigus disposés régulièrement. En vue apicale, l'hémisomate est à contour elliptique ; son sommet glabre est bordé de courtes épines.

Dimensions : cellule de $30 \times 22 \mu \mathrm{m}$, isthme de $7 \mu \mathrm{m}$ de largeur, épaisseur de $15 \mu \mathrm{m}$.

Distribution : Afrique : Mozambique, Niger

Cosmarium anthophorum Couté et Rousselin fig. 43, éch. : F 3/96

La cellule, plus longue que large, présente une constriction médiane profonde et un sinus fermé. Les hémisomates sont à contour régulièrement elliptique. La paroi cellulaire (sauf la région médiane) est ornée de verrues disposées régulièrement en quinconce. Chacune des verrues est bordée d'une colorette polygonale crénelée. La vue apicale montre le même type d'ornementation sur toute la surface. Par hémisomate, on a deux pyrénoïdes.

Dimensions : cellule de 71 x $58 \mu \mathrm{m}$, isthme de $16 \mu \mathrm{m}$ de largeur, épaisseur de 35 $\mu \mathrm{m}$.

Distribution : Afrique : Niger, Mozambique.

Cosmarium beatum W. et G.S. West fo. achrondrum Compère

fig. 44, F 9/97

La cellule a un contour plus ou moins régulièrement crénelé et un sinus ouvert, aigu. Les hémisomates sont trapézoïdaux. On observe deux pyrénoïdes par hémisomate. Nos exemplaires diffèrent de l'espèce-type par l'absence d'ornementation au centre de la paroi de l'hémisomate.

Dimensions : cellule de 34 x $32 \mu \mathrm{m}$; isthme de $9 \mu \mathrm{m}$; épaisseur de $10 \mu \mathrm{m}$.

Distribution : connu jusque là uniquement en Afrique. 
Cosmarium beatum W. et G.S. West var. apertum Couté et Rousselin

fig. 45, éch. : F 9/97

La cellule a un contour régulièrement crénelé et un sinus ouvert aigu. Les hémisomates sont trapézoïdaux et ornés chacun au centre d'une grosse verrue entourée d'une demi-couronne de trois verrues plus réduites.

Dimensions : cellule de 32 × $30 \mu \mathrm{m}$, isthme de $12 \mu \mathrm{m}$, épaisseur de $15 \mu \mathrm{m}$.

Distribution : en Afrique : Niger

\section{Cosmarium botrytis (Menegh.) Ralfs} fig. 46, éch. : B 8/97

[Syn. : Euastrum botrytis Ehrb.]

La cellule, plus longue que large à constriction médiane profonde, est caractérisée par un sinus étroitement linéaire mais dilaté à l'extrémité extérieure. Les hémisomates, trapézoïdaux, sont ornés de grosses verrues arrondies, réparties uniformément sur toute la surface de la paroi. Par hémisomate, on a deux pyrénoïdes. En vue apicale, la cellule est elliptique.

Dimensions : cellule de 86 x $59 \mu \mathrm{m}$, isthme large de $21 \mu \mathrm{m}$, épaisseur de $25 \mu \mathrm{m}$.

Distribution: cosmopolite. En Afrique : Afrique du Sud, Algérie, Côte d'Ivoire, Égypte, Kenya, Libye, Maroc, Mozambique, République Démocratique du Congo, Soudan, Tanzanie, Tchad, Tunisie.

Cosmarium connatum (Bréb.) Ralfs var. africanum Fritsch et Rich

fig. 47, éch.: F 11/95

[Syn.: Cosmarium connatum Bréb. ex Ralfs]

La cellule, plus longue que large, a une constriction médiane modérée, un sinus peu marqué et un isthme très large. Les hémisomates sont transversalement subelliptiques avec de larges bases. Les apex sont légèrement plats. En vue apicale, la cellule est circulaire ou largement elliptique. La paroi est finement ponctuée. Deux pyrénoïdes sont présents par hémisomate. On observe une rangée de pores de part et d'autre de l'isthme.

Dimensions : cellule de 58 x $41 \mu \mathrm{m}$, isthme large de $29 \mu \mathrm{m}$, épaisseur de $33 \mu \mathrm{m}$.

Distribution: cosmopolite. En Afrique: Afrique du Sud, Côte d'Ivoire, Guinée, Kenya, Madagascar, Malawi, Mali, Mozambique, Ouganda, République
Démocratique du Congo, Sierra Léone, Soudan, Sud-Ouest Africain, Tanzanie, Tchad, Zambie, Zimbabwé.

Cosmarium contractum Kirchner var. ellipsoideum (Elfv.) West et West

fig. 48, éch. : F 8/97

Petite cellule, très légèrement plus longue que large, à constriction médiane peu profonde et a sinus ouvert. Les hémisomates sont elliptiques. Chaque hémisomate renferme un pyrénoïde. En vue apicale, la cellule est plus largement elliptique.

Dimensions : cellule de $28 \times 24 \mu \mathrm{m}$, isthme large de $11 \mu \mathrm{m}$, épaisseur de $13 \mu \mathrm{m}$.

Distribution: cosmopolite. En Afrique, l'espèce est connue: d'Afrique du Sud, Algérie, Angola, Mali, Madagascar, Mozambique, Ouganda, Sierra Léone, Tanzanie, Sud-Ouest Africain, Tchad, Zambie.

Cosmarium contractum Kirchner var. pachydermum Scott et Prescott fig. 49, éch. : F 8/95

La cellule, plus longue que large, a une constriction médiane profonde, et un sinus ouvert, aigu. Les hémisomates sont largement ellipsoïdaux et contiennent chacun un pyrénoïde. Chaque hémisomate montre une tumeur médiane très marquée. La paroi est finement ponctuée.

Dimensions : cellule de 41 × $35 \mu \mathrm{m}$, isthme de $8 \mu \mathrm{m}$ de largeur, épaisseur de $23 \mu \mathrm{m}$.

Distribution: cosmopolite.

Cosmarium margaritatum (Lund.) Roy et Biss. fig. 50, éch. : B.9/97'

La cellule, large, a une constriction médiane profonde et un sinus étroitement linéaire avec une légère dilatation à l'extrémité. Les hémisomates sont subrectangulaires, convexes à l'apex et ornés de verrues arrondies, disposées régulièrement sur toute la surface. Chaque hémisomate renferme deux pyrénoïdes.

Dimensions : cellule de $80 \times 66 \mu \mathrm{m}$, isthme de $19 \mu \mathrm{m}$ de largeur, épaisseur de $38 \mu \mathrm{m}$.

Distribution: cosmopolite. En Afrique: Afrique du Sud, Malawi, Mali, Mozambique, Ouganda, Sierra Léone, Sud-Ouest Africain, Tchad, Zambie. 


\section{Cosmarium monodii Bourrelly}

fig. 51, éch.: F 8/95

La cellule, légèrement plus longue que large, a une constriction médiane profonde et un sinus ouvert, aigu. Les hémisomates, subtrapézoïdaux à subelliptiques, sont ornés, sur les côtés, de deux rangées de fortes épines. La région centrale de chaque hémisomate montre un épaississement interne qui, en surface, est orné de grosses scrobiculations. La paroi est finement ponctuée. On observe deux pyrénoïdes par hémisomate.

Dimensions : cellule de 85 x $81 \mu \mathrm{m}$, isthme large de $28 \mu \mathrm{m}$, épaisseur de $47 \mu \mathrm{m}$.

Distribution: Afrique: Guinée, Mali, Mozambique, Sud-Ouest Africain, Tchad, Zambie, Zimbabwé.

Cosmarium quadrum Lund. var. minus Nordst. fig. 52, éch. : F 11/96

Les cellules, plus longues que larges, ont une constriction médiane profonde et un sinus étroitement linéaire. Les hémisomates sont subrectangulaires, ornés sur toute leur surface de grosses verrues rondes. On observe deux pyrénoïdes par hémisomate. En vue apicale, les hémisomates sont oblongs-elliptiques avec des côtés parallèles. La variété diffère de l'espèce par ses faibles dimensions.

Dimensions: cellules de 46 x 39-42 $\mu \mathrm{m}$, isthme large de $11 \mu \mathrm{m}$, épaisseur de $25 \mu \mathrm{m}$

Distribution: l'espèce est cosmopolite. En Afrique: Afrique du Sud, Algérie, Égypte, Guinée Conakry, Kenya, Libye, Malawi, Ouganda, Soudan, République Démocratique du Congo, Sierra Léone, Tanzanie, Tchad, Zimbabwé.

\section{Cosmarium quasillus Lund. fo.} devonense Croasdale

fig. 53, éch. : F12/95

La cellule, plus longue que large, a une constriction médiane profonde et un sinus étroitement linéaire et légèrement dilaté vers l'extrémité. Les hémisomates sont pyramidaux, tronqués au sommet et à marge ondulée. Chaque hémisomate présente, au centre, une tumeur granulée. La paroi cellulaire est granulée avec des ponctuations entre les granules. En vue apicale, la cellule est légèrement elliptique.

Dimensions : cellule de 43 x $38 \mu \mathrm{m}$, isthme large de $10 \mu \mathrm{m}$, épaisseur de $20 \mu \mathrm{m}$.
Distribution : cosmopolite. En Afrique : Côte d'Ivoire, Mozambique, Tchad.

Cosmarium subhammeri Rich var. africanum Bourr.

fig. 54, éch. : B 8/97

Petite cellule, plus longue que large, à hémisomates trilobés, ornés sous l'apex de deux grosses papilles arrondies. A la base de chaque hémisomate et de chaque côté, on observe, une dent obtuse. Les apex sont plans et déprimés en leur milieu. La paroi est lisse. Un seul pyrénoïde est visible par hémisomate. Dimensions : cellule de $32 \times 27 \mu \mathrm{m}$, isthme de $6 \mu \mathrm{m}$ de largeur, épaisseur de $12 \mu \mathrm{m}$.

Distribution: Afrique tropicale et australe : Mali, Mozambique, Tchad.

Cosmarium venustum (Bréb.) Arch. fig. 55, éch. : B 8/97

Petite cellule, plus longue que large, à constriction médiane profonde et à sinus étroitement linéaire et dilaté à l'extrémité. Les hémisomates sont trapézoïdaux, à contour marqué de 5 larges ondulations et à apex concave. La paroi est lisse. Un seul pyrénoïde est visible par hémisomate.

Dimensions : cellule de 42 × $30 \mu \mathrm{m}$, isthme large de $12 \mu \mathrm{m}$, épaisseur de $13 \mu \mathrm{m}$.

Distribution: cosmopolite. En Afrique: Soudan, Tchad.

Euastrum platycerum Reinsch var. obtusius Grönbl. et Croas.

fig. 56, éch. : F 3/96

Les cellules ont des hémisomates trilobés et un sinus fermé. Le lobe apical est émarginé à presque plan. Les lobes latéraux sont émarginés latéralement. Tous les lobes sont ornés d'épines coniques. Chaque hémisomate a une tumeur centrale ornée de deux couronnes concentriques de verrues ;

Dimensions : cellules de $50 \times 42 \mu \mathrm{m}$; isthme large de $7 \mu \mathrm{m}$, lobe polaire large de $16 \mu \mathrm{m}$ Distribution : variété africaine (Mali).

Euastrum spinulosum Delponte var. lindae Grönblad et Scott fo. minor Couté \& Rousselin

fig. 57, éch. : F 3/96

La cellule, plus longue que large, a des hémisomates divisés chacun en cinq lobes. La marge du lobe apical est profondément déprimée dans sa partie centrale. Comme pour 
Euastrum spinulosum, notre forme présente des marges garnies de courtes épines. Chaque hémisomate possède une tumeur centrale. Le plaste pariétal a deux pyrénoïdes.

Dimensions : cellule de 66 x $57 \mu \mathrm{m}$, isthme large de $14 \mu \mathrm{m}$.

Distribution : Afrique (Mali)

Euastrum subalpinum Messik. var. crassum Messik.

fig. 58, éch. : F 12/97'

Petit Euastrum à contour quadrangulaire, plus long que large. La marge apicale de chaque hémisomate est marquée d'une incision largement ouverte. Les marges latérales sont déprimées. Le contour de la cellule est souligné de verrues coniques.

Dimensions : cellule de 18 x $13 \mu \mathrm{m}$, isthme large de $4 \mu \mathrm{m}$.

Distribution : Canada, Suisse, Venezuela. En Afrique: Tchad.

Micrasterias pinnatifida (Kütz.) Ralfs fig. 59, éch. : F 5/95

La cellule, plus large que longue, est profondément constrictée et a un sinus ouvert. Les lobes latéraux uniques, sont coniques, horizontalement étendus et bifides aux extrémités. Le lobe polaire est généralement horizontal et étalé, avec un apex légèrement convexe et déprimé dans son milieu. Il est moins étendu que les lobes latéraux et aussi bifides aux extrémités. Le chloroplaste renferme de nombreux petits pyrénoïdes. La paroi est finement ponctuée.

Dimensions : cellule de 60 x $83 \mu \mathrm{m}$, isthme de $8 \mu \mathrm{m}$ de largeur.

Distribution: forme de régions chaudes. En Afrique : Mali

Pleurotaenium trabecula (Ehr.) Näg. fig.60, éch. : B 8/97'

Les cellules en forme de bâtonnets cylindriques, rectilignes, montrent un renflement juste avant l'isthme et des apex tronqués. La paroi est lisse. Les plastes, en rubans pariétaux parallèles, renferment chacun une série de pyrénoïdes.

Dimensions : cellules de 136-148 x 8-10 $\mu \mathrm{m}$. Distribution : cosmopolite. En Afrique : Côte d'Ivoire, Mozambique.

Staurastrum anatinum Cooke et Wills fo.

fig. 61, éch.: F 12/95
Les cellules, plus larges que longues (avec les bras), ont un sinus ouvert. Les hémisomates sont cupiliformes. Chaque hémisomate a 3 angles prolongés chacun en un bras ayant un nombre modéré d'ondulations (4-5) et terminé par 3 épines. La marge apicale est convexe ; on y observe des verrues sous-apicales. Cette forme se distingue de l'espèce type par son absence d'ornementation apicale.

Dimensions : cellules de 26 x 51-56 $\mu \mathrm{m}$ (avec les bras), isthme large de $8 \mu \mathrm{m}$.

Distribution: cosmopolite. En Afrique : Afrique du Sud, Kenya, Ouganda, Sierra Léone, Tanzanie, Tchad.

\section{Staurastrum bieneanum Rabh.} fig. 62 , éch. : B 12/97

La cellule a un sinus ouvert, aigu. En vue frontale, l'hémisomate est ellipsoïdal et triangulaire en vue apicale avec des angles subaigus et des marges légèrement déprimées. La paroi cellulaire est lisse. Un plaste pariétal à un pyrénoïde occupe chaque hémisomate.

Dimensions : cellule de 35 × $35 \mu \mathrm{m}$, isthme de $9 \mu \mathrm{m}$ de largeur.

Distribution: cosmopolite. En Afrique : Madagascar, Tanzanie, Tchad.

Staurastrum cyclacanthum W. et G. S. West fig. 63, éch.: F 2/97

Les cellules, triangulaires en vue apicale, ont des angles prolongés chacun en un bras. Les hémisomates sont ornés de verrues à l'apex et de deux appendices verticaux ou obliques à la naissance de chaque bras. En vue apicale, la cellule de contour triangulaire, montre un cercle de 6 verrues trifides et une paire de verrues bifides à la base de chaque bras.

Dimensions : cellules de 33 x 59-63 $\mu \mathrm{m}$ (avec les bras).

Distribution: pantropicale. En Afrique: Mozambique, Ouganda, Soudan, Tchad.

Staurastrum floriferum W. et G. S. West fig. 64, éch.: F 2/97

Petite cellule, plus longue que large (avec les bras), à sinus ouvert et à isthme relativement large. Les hémisomates, cupiliformes, à marges latérales convexes, se prolongent aux angles par des bras horizontaux terminés par des épines. La marge apicale a deux verrues. En vue apicale on observe une couronne de 6 verrues bifides. 
Dimensions : cellules de 27 x 40-42 $\mu \mathrm{m}$ (avec les bras), isthme de $7 \mu \mathrm{m}$ de largeur.

Distribution: cosmopolite. En Afrique: Ouganda, Sierra Léone, Soudan, Tchad, Zambie.

Staurastrum leptocladum Nordst. var. coronatum Scott et Grönbl.

fig. 65 , éch. : F 7/95

Les cellules, plus larges que longues (avec les bras), sont légèrement circulaires en vue apicale et longuement prolongées de chaque côté par un long bras terminé par deux dents. Le corps de l'hémisomate est renflé et orné d'une rangée d'épines au-dessus de l'isthme. L'apex de chaque hémisomate est pourvu de deux cornes disposées de façon divergente. Dimension : cellules de 30-34 x $88 \mu \mathrm{m}$ (avec les bras), isthme de $7 \mu \mathrm{m}$ de largeur.

Distribution : Afrique : Côte d'Ivoire, Tchad.

\section{Staurastrum margaritaceum (Ehr.) Ralfs var. gracilius Scott et Grönbl.} fig. 66, éch.: B 7/97'

Petites cellules relativement plus longues que larges, quadriradiées, à bras courts, divergents. Le sinus est ouvert et l'isthme large. La base des hémisomates est ornée d'une rangée de petites verrues. Chaque hémisomate a un plaste avec un pyrénoïde.

Dimensions : cellule de 21 x 19-20 $\mu \mathrm{m}$, isthme de $5 \mu \mathrm{m}$.

Staurastrum senarium (Ehr.) Ralfs fig. 67 , éch. : F 8/95

Cellule, relativement plus large que longue, à constriction médiane profonde, à sinus ouvert et à isthme large. L'hémisomate est fusiforme. Les marges latérales convexes, divergent directement du sinus vers les angles apicaux qui se prologent en de courts bras bifides. Les bras forment une spire de 6 autour de l'hémisomate.

En vue apicale, la cellule est triangulaire, la marge montrant deux verrues de chaque côté, en regard avec les verrues intramarginales.

Dimensions : cellule de 31 × $32 \mu \mathrm{m}$ (avec les bras), isthme de $5 \mu \mathrm{m}$ de largeur.

Staurastrum tetracerum Ralfs var. subexcavatum Grönbl.

fig. 68, éch. : F 8/95

Les cellules, ont des hémisomates tordus l'un par rapport à l'autre. Chaque hémisomate se prolonge de chaque côté en un bras divergent relativement long, denticulé sur toute sa longueur. L'apex est profondément concave.

Dimensions : cellules de 25-35 x 39-40 $\mu \mathrm{m}$ (avec les bras), isthme de $6 \mu \mathrm{m}$ de largeur.

Distribution : cosmopolite. En Afrique : Côte d'Ivoire, Tchad, Zimbabwé.

Staurodesmus psilosporus (Nordst. et Löfgr.) Teiling var. retusus (Grönb.) Teiling fig. 69, éch.: F 5/97

Les cellules ont des hémisomates trapézoïdaux, à apex incurvé, dont les extrémités s'étirent en de courtes épines divergentes.

Dimensions : cellules de 12-14 x $13 \mu \mathrm{m}$ (sans les épines), isthme de $5 \mu \mathrm{m}$ de largeur, épines de $5 \mu \mathrm{m}$ de longueur.

Distribution: Afrique: Soudan, Tchad,. Amérique.

Staurodesmus subtriangularis (Borge) Teiling fig. 70, éch. : F 8/95

Cellules à hémisomates triangulaires en vue de face. Les marges latérales sont légèrement constrictées au-dessus de l'isthme et l'apex est concave, mais déprimé au milieu. Chaque hémisomate est prolongé, à chaque extrémité, par une longue épine divergente.

Dimensions: cellules de 28-30 x $23 \mu \mathrm{m}$, isthme large de $5 \mu \mathrm{m}$, épines de 12-14 $\mu \mathrm{m}$ de longueur.

Distribution: cosmopolite. En Afrique: Afrique du Sud, Algérie, Côte d'Ivoire, Kenya, Mali, Ouganda, Tanzanie, Tchad.

\section{Staurodesmus triangularis (Lagerh.) Teiling fig. 71, éch. : F 11/96}

Les cellules, à hémisomates triangulaires en vue frontale, se prolongent à chaque extrémité par une épine convergente. Elles ont un sinus ouvert.

Dimensions: cellules de 22-24 x $22 \mu \mathrm{m}$ (sans les épines), isthme large de $4 \mu \mathrm{m}$, épines longues de 9-12 $\mu \mathrm{m}$.

Distribution: cosmopolite. En Afrique: Afrique du Sud, Algérie, Côte d'Ivoire, Kenya, Mali, Ouganda, Tanzanie, Tchad.

Xanthidium subtrilobum W. et G.S. West var. africanum (Schmidle) Grönbl. et Scott

fig. 72, éch. : F 5/95

Les cellules ont des hémisomates trilobés, un sinus ouvert. Le lobe apical est pourvu de 4 
épines dressées, subverticales, et les lobes latéraux sont, quant à eux, pourvus chacun de 3-4 épines. Chaque lobe latéral possède à sa base 1-2 verrues. Chaque hémisomate possède, en son centre, une protubérance ornée d'une couronne de verrues.

Dimensions : cellule de 70 x $60 \mu \mathrm{m}$ (sans les épines), isthme de $8 \mu \mathrm{m}$ de largeur.

Distribution: Afrique tropicale: Burkina Faso, Côte d'Ivoire, Guinée, Mali, Mozambique, Soudan, Tchad, Zimbabwé.

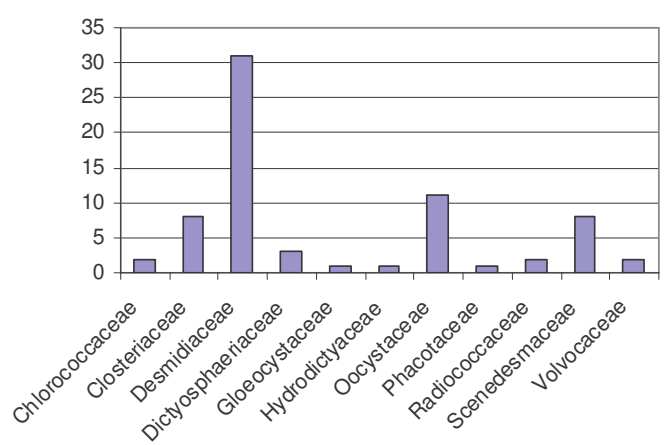

Figure 2 : Nombre d'espèces par famille

Tableau 2 : Liste des taxa cités

\begin{tabular}{|c|c|c|}
\hline Embranchements & Familles & Espèces \\
\hline \multirow[t]{35}{*}{ Chlorophyta } & \multirow[t]{2}{*}{ Chlorococcaceae } & $\begin{array}{l}\text { Tetraedron caudatum (Corda) Hansg. var. incisum (Lagerh.) } \\
\text { Brunth. }\end{array}$ \\
\hline & & T. cruciatum (Wall.) G.S. West \\
\hline & \multirow[t]{3}{*}{ Dictyosphaeriaceae } & Botryococcus braunii Kütz. \\
\hline & & Dimorphococcus lunatus A. Braun \\
\hline & & Westella botryoides (West) De Wild. \\
\hline & Hydrodictyaceae & Pediastrum biradiatum Meyen var. longecornutum Gutw. \\
\hline & \multirow{11}{*}{ Oocystaceae } & Chodatella quadriseta Lemm. \\
\hline & & C. subsalsa Lemm. \\
\hline & & Monoraphidium griffithii (Brek.) Kom.-Legner \\
\hline & & Echinosphaerella limnetica G.M. Smith \\
\hline & & Kirchneriella obtusa (Korsh.) Kom. \\
\hline & & Monoraphidium indicum Hind. \\
\hline & & Nephrocytium limneticum (G.M. Smith) G.M. Smith \\
\hline & & N. lunatum W. West \\
\hline & & N. obesum W. et G.S. West \\
\hline & & Oocystis crassa Wittr. \\
\hline & & Treubaria eurycantha (Schmidle) Korsch. \\
\hline & \multirow{2}{*}{ Radiococcaceae } & Coenochloris pseudoquadriguloides Tell \\
\hline & & C. pyrenoidosa Korsch. \\
\hline & \multirow{8}{*}{ Scenedesmaceae } & Actinastrum aciculare Playf. fo. africanum Compère \\
\hline & & A. hantzschii Lagerh. var. fluviatile Schröder \\
\hline & & Coelastrum cambricum Arch. var. intermedium (Bohl.) G.S. West \\
\hline & & C. pseudomicroporum Korsch. \\
\hline & & Crucigenia crucifera (Wolle) Collins \\
\hline & & Scenedesmus balatonicus Hortob. \\
\hline & & S. obtusus Meyen fo. ecornis (Ehr.) Compère \\
\hline & & Tetrallantos lagerheimii Teil \\
\hline & Gloeocystaceae & Gloeocystis ampla (Kütz.) Rabenh. \\
\hline & Phacotaceae & Wislouchiella planctonica Skvortz. \\
\hline & \multirow{2}{*}{ Volvocaceae } & Gonium pectorale Müll. \\
\hline & & G. sociale (Duj.) Warming \\
\hline & \multirow{4}{*}{ Closteriaceae } & Closterium acerosum (Schrank) Ehr. var. borgei (Borge) Krieg. \\
\hline & & C. acerosum (Schrank) Ehr. var. minus Hantzsch \\
\hline & & C. acutum de Bréb. Ex Ralfs var. variabile (Lemm.) Krieg. \\
\hline & & C. dianae Ralfs var. brevius (Petk.) Krieg. \\
\hline
\end{tabular}




\begin{tabular}{|c|c|}
\hline \multirow[t]{27}{*}{ Desmidiaceae } & C. botrytis (Menegh.) Ralfs \\
\hline & C. connatum (Bréb.) Ralfs var. africanum Fritsch et Rich \\
\hline & C. contractum Kirchn. var. ellipsoideum (Elfv.) W et G. S. West \\
\hline & C. contractum Kirchn var. pachydermum Scott et Prescott \\
\hline & C. margaritatum (Lund.) Roy et Biss. \\
\hline & C. monodii Bourrelly \\
\hline & C. quadrum Lund. var. minus Nordst. \\
\hline & C. quasillus Lund. fo. devonense Croasdale \\
\hline & C. subhammeri Rich var. africanum Bourr. \\
\hline & C. venustum (Bréb.) Arch. \\
\hline & Euastrum platycerum Reinsch var. obtusius Grönbl. et Croas. \\
\hline & $\begin{array}{l}\text { E. spinulosum Delp. var. lindae Grönbl. et Scott fo. minor Couté } \\
\text { et Rousselin }\end{array}$ \\
\hline & E. subalpinum Messik. var. crassum Messik. \\
\hline & Micrasterias pinnatifida (Kütz.) Ralfs \\
\hline & Pleurotaenium trabecula (Ehr.) Näg. \\
\hline & Staurastrum anatinium Cooke et Wills fo. \\
\hline & S. bieneanum Rabh. \\
\hline & S. cyclacanthum W et G.S West \\
\hline & S. floriferum W et G. S West \\
\hline & S. leptocladum Nordst. Var. coronatum Scott et Grönbl. \\
\hline & S. margaritaceum (Ehr.) Ralfs var. gracilius Scott et Grönbl. \\
\hline & S. senarium (Ehr.) Ralfs \\
\hline & S. tetracerum Ralfs var. subexcavatum Grönbl. \\
\hline & S. psilosporus (Nordst. et Löfgr.) var. retusus Grönbl. et Teil. \\
\hline & S. subtriangularis (Borge) Teiling \\
\hline & S. triangularis (Lagerh.) Teiling \\
\hline & $\begin{array}{l}\text { Xanthidium subtrilobum W. et G.S. West var. africanum } \\
\text { (Schmidle) Grönbl. et Scott }\end{array}$ \\
\hline
\end{tabular}

\section{DISCUSSION}

Un nombre assez élevé de nouveaux taxa a été rencontré dans l'embranchement des Chlorophyta (Tableau 2). Cela indique que les conditions physico-chimiques du réservoir de Bagré sont beaucoup plus favorables au développement des microalgues appartenant à cet embranchement. Les espèces les mieux favorisées dans ce groupe appartiennent à la famille des Desmidiaceae qui représente près de la moitié du nombre d'espèces des 11 familles. Cela explique la capacité des espèces de la famille à s'adapter et à se développer dans des conditions physico-chimiques et environnementales diverses. Selon Bourrelly (1990), les Desmidiaceae ont une grande importance dans la question d'écologie; comme les Diatomées elles sont très sensibles aux variations de la composition chimique de l'eau et à la température. Selon cet auteur, toute une série d'espèces de cette famille est liée aux eaux alcalines. C'est le cas dans notre étude où la majorité des espèces nouvelles de
Chlorophyta appartiennent à la famille des Desmidiaceae. Le réservoir étant un milieu alcalin favorise sans doute, le développement des espèces de cette classe.

Le développement des espèces dans les écosystèmes aquatiques nécessite un certain nombre de paramètres dont elles sont adaptées. L'oxygène dissout nécessaire pour la respiration, l'azote et le phosphore sont surtout des paramètres dont les variations influencent la diversité et la structure de la communauté algale dans un milieu aquatique. Ainsi, selon Dussart (1992), l'azote est nécessaire par exemple pour le genre Scenedesmus. Quant au phosphore, les algues sont capables de l'extraire de l'eau même quand il $\mathrm{n}$ y'a que très peu (Dussart, 1992). Cela indique la place et la nécessité de ces minéraux dans le cycle de vie des microalgues. Dans cette étude, le tableau 1 montre une présence plus ou moins importante des minéraux dans le réservoir. La présence de ces minéraux favorables (azote et phosphate) en interactions avec les autres 


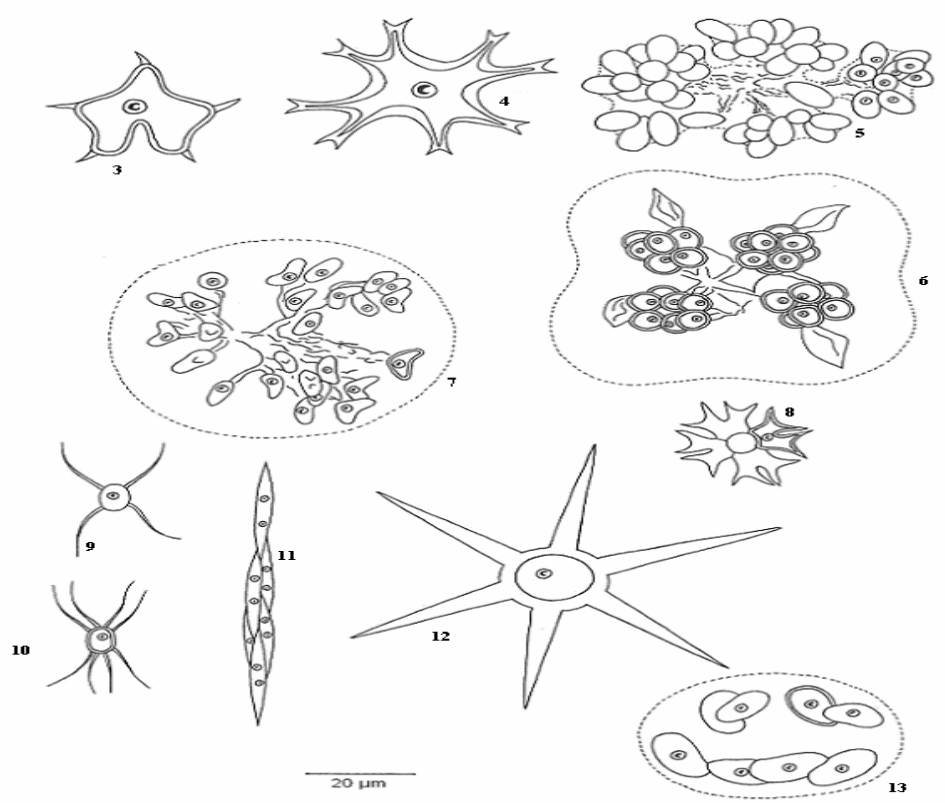

Fig.3-13: 3: Tetraedron caudatum var incisum ; 4 : T. cruciatum, 5 : Botryococcus braunii ; 6 : Dimorphococcus lunatus ; 7 : Westella botryoides ; 8 : Pediastrum biradiatum var. longecornutum ; 9: Chodatella quadriseta, $10:$ C. subsalsa ; 11 : Monoraphidium griffithii ; 12 : Echinosphaerella limnetica ; 13 : Kirchneriella. obtusa.

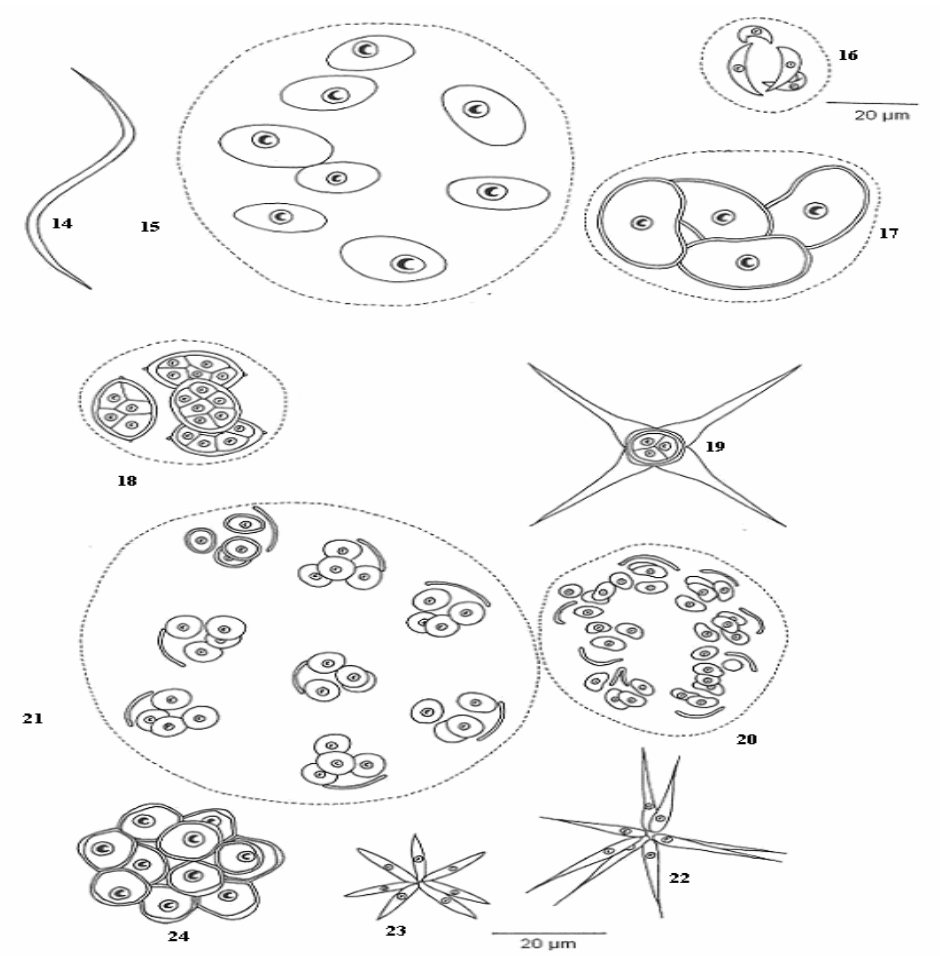

Fig. 14-24: 14 : Monoraphidium indicum; 15 : Nephrocytium. limneticum; $16:$ N. lunatum, $17: N$. obtusum; 18: Oocystis crassa; 19: Treubaria eurychanta; 20: Coenochloris pseudoquadrigulö̈des; 21 : C. pyrenö̈dosa; 22 : Actinastrum acicularis fo. africanum; 23 : A. hantzschii var. fluviatile; 24 : Coelastrum cambricum var. intermedium. 

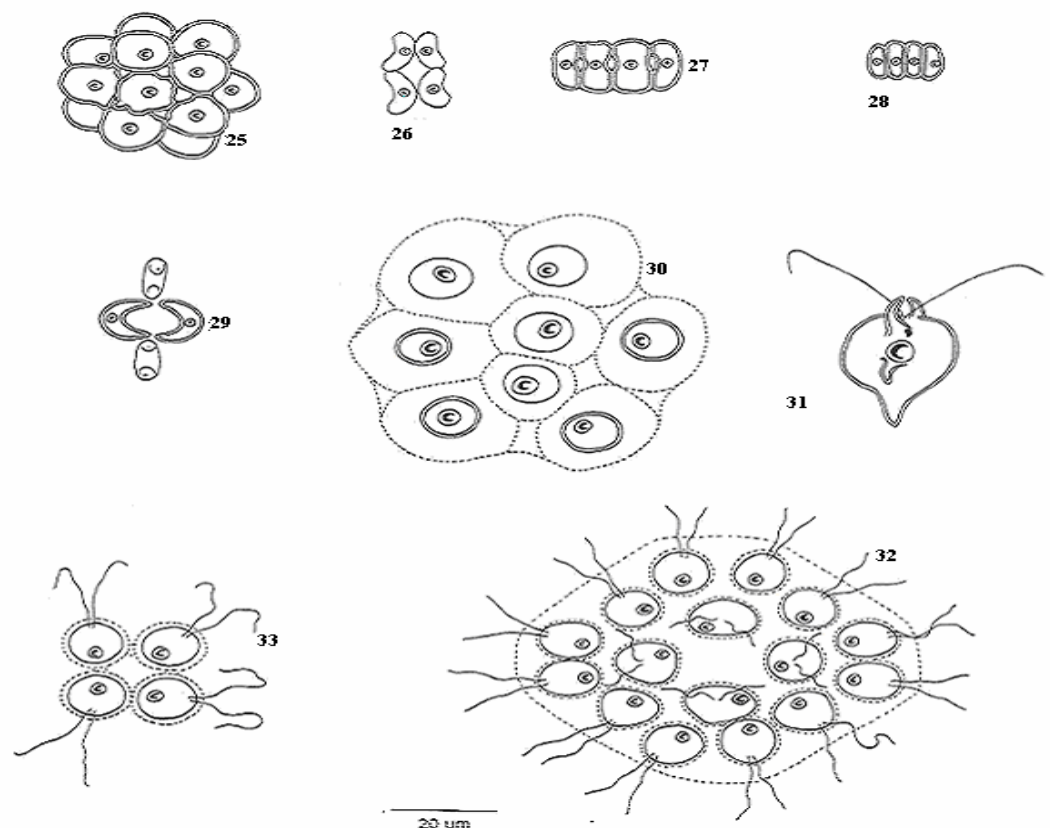

Fig. 25-33: 25 : Coelastrum. pseudomicroporum; 26 : Crucigenia crucifera ; 27 : Scenedesmus balatonicus ; 28 : S. obtusus fo. ecornis ; 29 : Tetrallantos lagerheimii ; 30 : Gloeocystis ampla ; 31 : Wislouchiella planctonica ; 32 : Gonium pectorale ; 33 : G. sociale
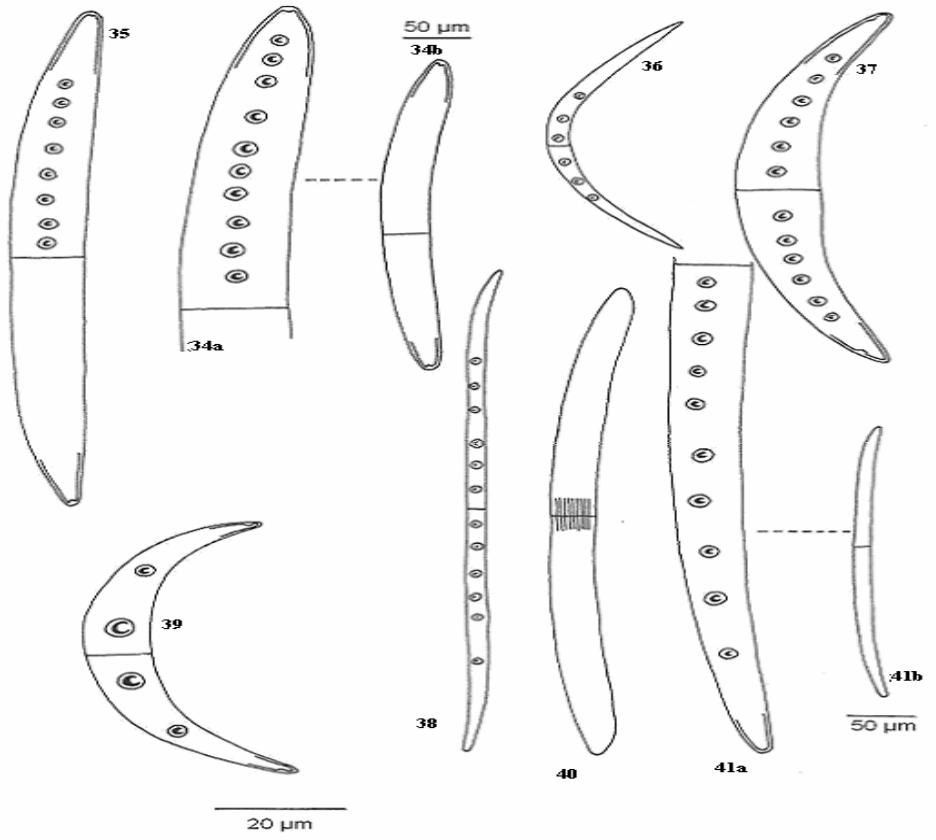

Fig. 34-41: 34 : Closterium acerosum var. borgei ; $35:$ C. acerosum var. minus ; $36:$ C. acutum var. variabile; 37 : C. dianae var. brevius ; 38: C. limneticum; 39: C. parvulum; $40:$ C. praelongum $; 41:$ C. toxon. 


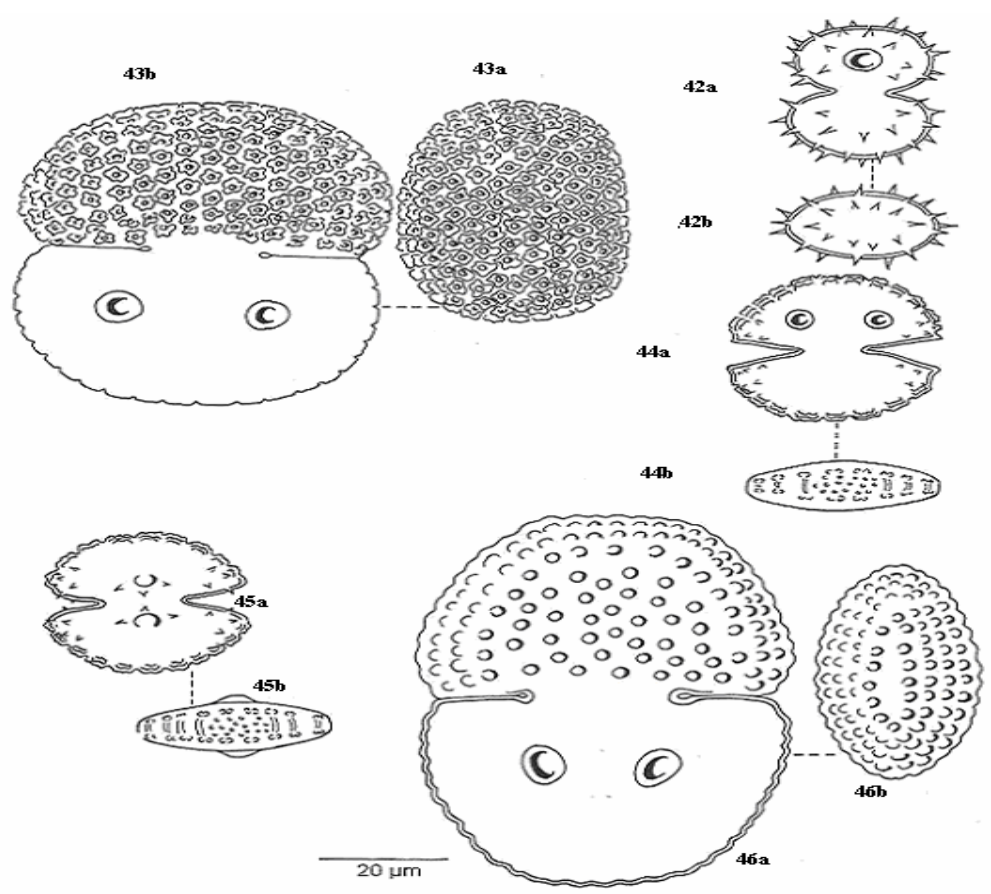

Fig. 42-46: 42 : Cosmarium aculeatum var. africanum (a : vue frontale, b : vue apicale), 43 : $C$. anthophorum (a: vue frontale b: vue apicale) ; $44: C$. beatum fo. achondrum; $45: C$. beatum var.apertum (a : vue frontale, $\mathrm{b}:$ vue apicale) ; $46: C$. botrytis (a : vue frontale b : vue apicale).

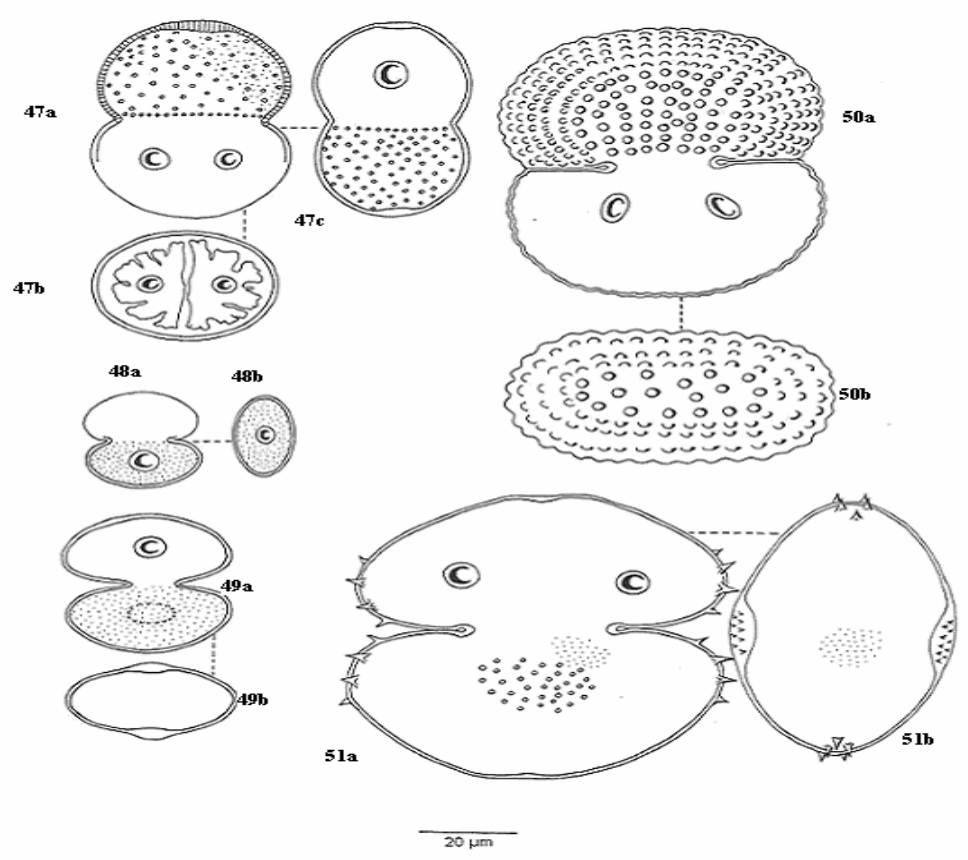

Fig. 47-51: 47 : Cosmarium connatum var. africanum (a: vue frontale, b : vue apicale); $48: C$. contractum var. ellipsö̈deum (a: vue frontale, b: vue apicale); 49: C. contractum var. pachydermum (a : vue frontale, b : vue apicale); $50: C$. margaritatum (a : vue frontale, b: vue apicale); 51 : C. monodii (a : vue frontale, b : vue apicale). 


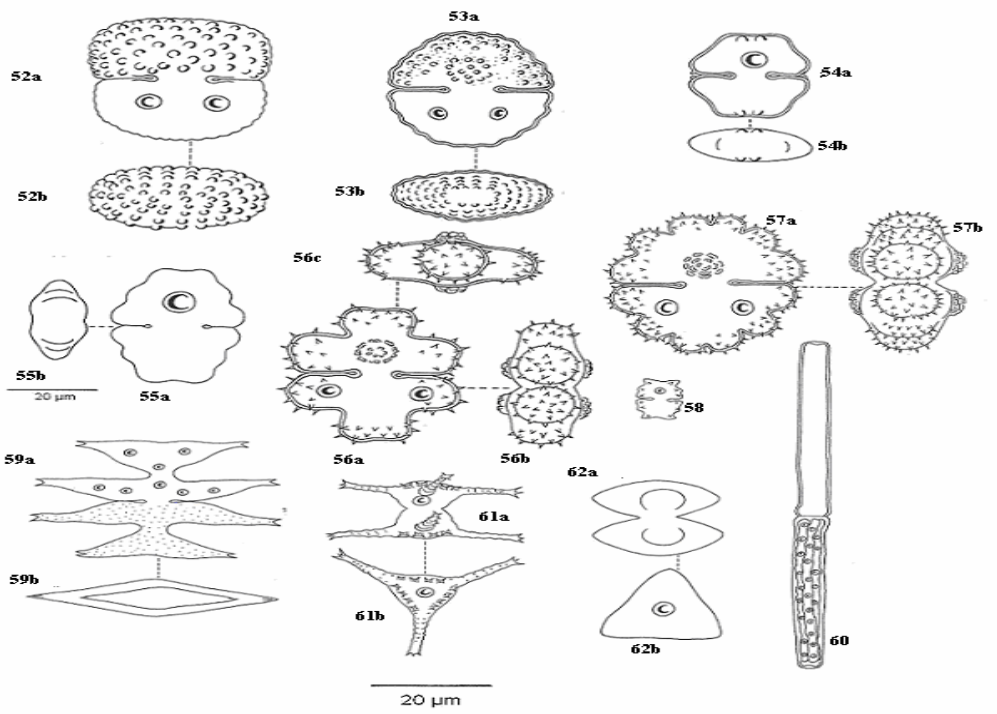

Fig. 52-62: 52 : C. quadrum var. minus (a : vue frontale, b : vue apicale); 53 : C. quasilus fo. devonense (a : vue frontale, $\mathrm{b}:$ vue apicale); $54:$ C. subhammeri var. africanum (a : vue frontale, $\mathrm{b}$ : vue apicale); $55:$ C. venustum (a: vue frontale, b: vue apicale); $56:$ Euastrum platycerum var. obtusius (a : vue frontale, b : vue de profil, c : vue apicale); 57 : E. spinulosum var. lindae fo minor (a: vue frontale, b: vue apicale); 58: E. subalpinum var. crassum; 59: Micrasterias pinnatifida (a: vue frontale, b:vue apicale); 60: Pleurotaenium trabecula; 61 : Staurastrum anatinium (a : vue frontale, b : vue apicale); $62:$ S. bieneanum (a : vue frontale, b : vue apicale).

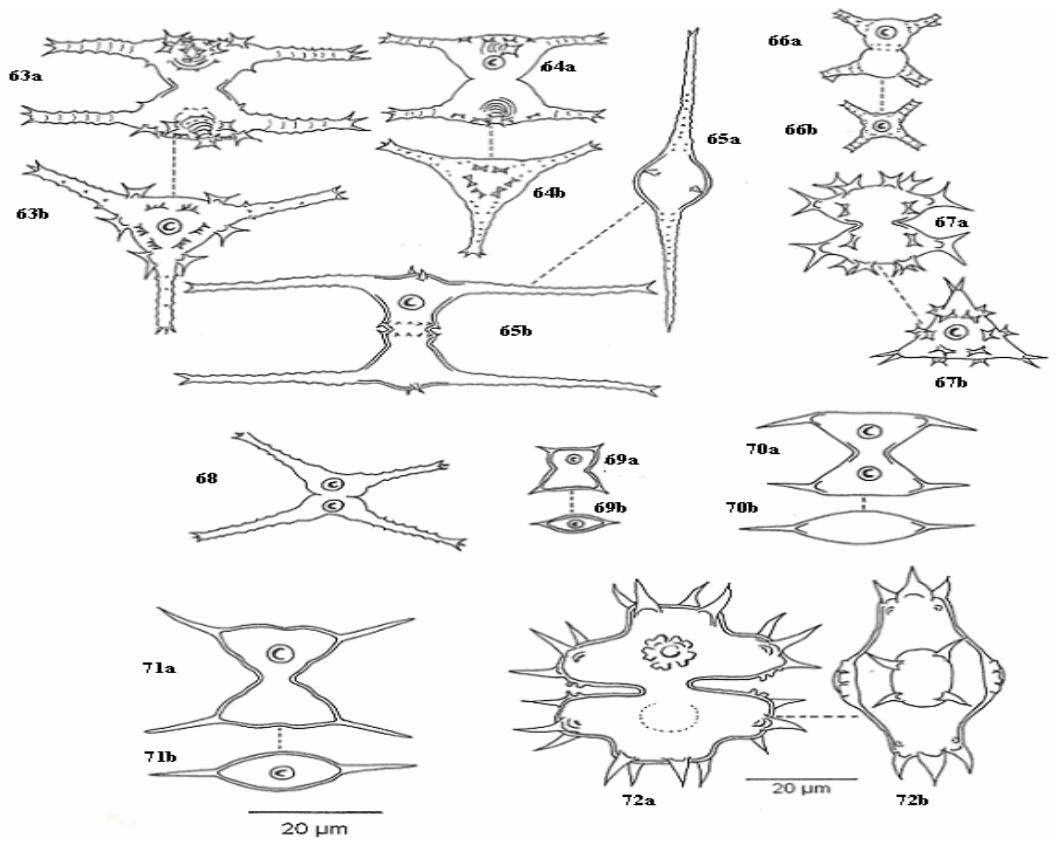

Fig. 63-72: 63 : Staurastrum cyclacanthum (a : vue frontale, b : vue apicale); 64 : S. floriferum (a : vue frontale, b : vue apicale) ; $65:$ S. leptocladum var. coronatum (a : vue frontale, b : vue apicale); $66:$ S. margaritaceum var. gracilius (a : vue frontale, b : vue apicale) ; $67:$ S. senarium (a : vue frontale, b : vue apicale), 68 : S. tetracerum var. subexcavatum ; 69 : S. psilosporus var. retusus (a : vue frontale, b: vue apicale); $70: S$. subtriangularis (a : vue frontale, b: vue apicale); $71: S$. triangularis (a : vue frontale $\mathrm{b}$ : vue apicale); 72 : Xanthidium subtrilobum var. africanum (a : vue frontale, $\mathrm{b}$ : vue apicale). 
facteurs physico-chimiques indispensables de l'eau du réservoir entraîne une diversité des espèces dans le milieu.

Aussi, d'un milieu à l'autre les conditions physico-chimiques et environnementales sont différentes. Ces différences observées sont à l'origine de différences d'espèces observées dans les milieux. Les nouveaux taxa rencontrés marquent alors une différence des conditions de vie des espèces du réservoir de Bagré par rapport aux conditions des milieux d'étude précédents au Burkina Faso.

Sur le plan biogéographique, parmi les espèces rencontrées, 62,85\% sont cosmopolites et se rencontrent dans diverses régions dans le monde; $5,71 \%$ sont subcosmoplites et retrouvées dans certaines régions du monde. Les espèces tropicales représentent environ $31,43 \%$ de l'ensemble. Ces espèces sont rencontrées dans les régions tropicales chaudes, soit en Afrique, en Asie ou en Amérique. Les formes cosmopolites sont les plus nombreuses d'où la capacité d'un grand nombre à s'adapter en même temps aux régions froides et aux régions chaudes. Cela est conforme aux résultats des travaux de Bourrelly (1990) qui montrent par exemple que dans la famille des Desmidiaceae, les formes cosmopolites sont très nombreuses.

\section{Conclusion}

$\mathrm{Au}$ terme de cette étude, 70 taxa de micro-algues (Chlorophyta), nouveaux pour le Burkina Faso ont été inventoriés et décrits. Ils se répartissent dans 11 familles et 29 genres. Les Desmidiaceae sont numériquement les plus abondantes, puis viennent les Oocystaceae, les Scenedesmaceae en égalité avec les Closteriaceae, les Dictyosphaeriaceae, les Chlorococcaceae qui elles sont en égalité avec les Radiococcaceae et Volvocaceae et enfin les Hydrodictyaceae, les Gloeocystaceae et les Phacotaceae numeriquement très faible ( $1,4 \%$ des espèces). 70 taxa nouveaux rencontrés montrent que le réservoir de Bagré est riche en espèces de micro-algues particulièrement celles des chlorophyta.

\section{REMERCIEMENTS}

Les auteurs remercient: le projet RUG/ÉCOLOGIE, pour son aide financier et logistique; le gouvernement Français, la
Fondation Charles de Gaulle et la Fondation Professeur Athanase Saccas sous l'égide de la Fondation de France, pour leur aide financière apportée à l'un des auteurs (F. ZONGO) .

\section{BIBLIOGRAPHIE}

Bourrelly P. 1990. Les Algues d'Eau Douce. Initiation à la Systématique. Tome I : les Algues Vertes. Ed. Boubée: Paris; 511p.

Da KP, Zongo F, Mascarell G, Couté A. 2004. Bagredinium, un nouveau genre de Péridiniales (Dinophyta) d'eau douce de l'Afrique de l'Ouest. Algological Studies, 111 : 45-61.

Gauthier-Lièvre L. 1964. Oedogoniacées Africaines. Nova Hedwigia, VII : 151558, 104 plches.

Gauthier-Lièvre L. 1965. Zygnématacées Africaines. Chr. Belser, Stuttgart and Wolf: Heppenheim; 210 p, 123 plches.

Hiddinga A. 1981. Desmidiacën iut de omgeving van Ouagadougou (Opper Volta). Interne rapporten van het Hugo de Vries Laboratorium, 1-62.

Huber-Pestalozzi G. 1961. Das Phytoplankton des Süsswassers. 5. Teil, Chlorophyceae (Grünalgen). Ord.: Volvocales. Stuttgart, $744 \mathrm{p}$.

Roman B. 1977. Étude du plancton des retenues d'eau alimentant la ville de Ouagadougou (Haute-Volta). Notes et Documents Voltaïques, 11: 1-52.

Roman B. 1979. Étude du plancton des retenues d'eau alimentant la ville de Ouagadougou (Haute-Volta). Notes et Documents Voltaïques, 13 : 50-106.

Zerbo P. 2004. Contribution à l'étude de la diversité du phytoplancton d'eau douce : Cas du réservoir de Loumbila, Burkina Faso. Mém. De Diplôme d'Études Approfondies, Université de Ouagadougou, $51 \mathrm{p}$.

Zongo B. 2007. Étude du phytoplancton dans le canal du parc Bangr-wéoogo (Ouagadougou/Burkina Faso). Mém de D.É.A., Université de Ouagadougou, 68p.

Zongo F. 1994. Contribution à l'étude du phytoplancton d'eau douce du Burkina Faso: cas du barrage $\mathrm{n}^{\circ} 3$ de la ville de Ouagadougou. Thèse de doctorat 3è cycle. Université de Ouagadougou, 170 p., 21 tabl. 11 fig., 23 plches.

Zongo F, Guinko S. 1999a. Quelques Chlorophyta et Euglenophyta du Barrage 
$\mathrm{n}^{\circ} 3$ de la ville de Ouagadougou (Burkina Faso). Annales de l'Université de Ouagadougou, sér. B, 7: 137-157.

Zongo F, Guinko S. 1999b. Quelques algues du réservoir hydroélectrique et hydroagricole de Bagré (Province du Boulgou/Burkina Faso). Bull. Jard. Bot. Nat. Belg., 67: 231-257.

Zongo F, Guinko S. 1999c. Flore algale du Burkina Faso (Afrique de l'Ouest). Revue Sciences et Technique, Sciences naturelles, 23(2): 147-171.
Zongo F, Guinko S. 2001. Nouvelles espèces d'algues d'eau douce au Burkina Faso. Annales de Botanique de l'Afrique de l'Ouest, 0(0): 1-6.

Zongo F, Mascarell G, Couté A. 2006. Strombomonas guinkoi sp. Nova (Euglenophyta), une nouvelle Euglenophyceae (Euglenophyta) d'eau douce du Burkina Faso (Afrique de l'Ouest). Algological Studies, 119: 17-27. 